\title{
CAMPYLOBACTERS TERMOTOLERANTES EN AVES DE CORRAL DE LA CIUDAD DE IQUITOS
}

(THERMOTOLERANT CAMPYLOBACTERS IN POULTRY FROM IQUITOS CITY)

Tresierra-Ayala, Alvaro*

Bendayan Acosta, Maria. E*

Bernuy Rodriguez, Alfonso*

Pereyra Panduro, Gustavo*

Espinoza Campos, Freddy*

\section{RESUMEN}

Con el fin de determinar la importancia de las aves de corral como reservorios naturales de los clásicos Campylobacters termotolerantes, nuestras fecales de pollos, pavos, patos y palomas de lquitos, fueron colocadas en un medio de enriquecimiento. Técnicas convencionales fueron utilizadas para identificar a $C$. jejuni ssp. jejuni, C. coli y $C$. lari.

Campylobacter spp. fueron aislados del $36,8 \%$ de los animales estudiados, aislándose con mayor frecuencia a $C$. jejuni ssp. jejuni $(23,6 \%)$. El pollo resultó ser el reservorio más importante de estos microorganismos $(54,0 \%)$.

Palabras claves: Campylobacter, termotolerantes, aves, reservorios.

\section{ABSTRACT}

In order to determine the importance of poultry as natural reservoirs of the classical thermotolerant Campylobacters; fecal samples from chickens, turkeys, ducks and pigeons in lquitos city were placed into an enrichment medium. Conventional techniques were used to identify $C$. jejuni ssp. jejuni, $C$. coli and $C$. lari.

Campylobacter spp. were isolated from $36,8 \%$ of the animals studied, being $C$. jejuni ssp. jejuni the most frequent $(23,6 \%)$. Chicken was the most important reservoir of these microorganisms $(54,0 \%)$.

Dpto. Acad. de Microbiología. Fac. de Ciencias Biológicas-UNAP, A partado Postal 751. IQUITOS-PERU. 


\section{INTRODUCCION}

Entre la variedad de agentes microbianos causantes de enteritis destacan las clásicas bacterias termotolerantes del género Campylobacter (C. jejuni ssp. jejuni, C. coli y C. lari) de la recientemente propuesta familia Campylobacteriaceae Vandamme y De Ley, (1991), bacterias zoonóticas que están emergiendo como enteropatógenos, con una frecuencia cada vez mayor, sobre todo en los países en vías de desarrollo (Soza et.al., 1987).

Estos microorganismos han sido aislados de una gran variedad de animales domésticos y silvestres Blaser et.al., (1980); Lazarte y Pacheco, (1986); Zamora et.al., (1992), siendo las aves de corral, los reservorios de mayor importancia, las cuales pueden constituir la fuente de contaminación para el hombre, otros animales, alimentos y cuerpos de agua Blaser et.al., (1983); Smibert, (1984).

En este sentido, teniendo en consideración que en nuestra región coexisten muchos factores ecológicos (alta temperatura y humedad, deficientes condiciones sanitarias, presencia de posibles reservorios, etc.), que podrían facilitar la transmisión de estos agentes, consideramos que el conocimiento que se tenga de la distribución de estas bacterias, podría ser un dato epidemiológico que contribuiría a mejorar las medidas de prevención y control de esta zoonosis.

\section{MATERIAL Y METODOS}

Muestras fecales tomadas al azar de 100 pollos, 50 pavos, 50 patos y 50 palomas criadas en la ciudad de lquitos (Perú), fueron obtenidas mediante la técnica del hisopado cloacal e inmediatamente colocadas en un medio de transporte y enriquecimiento selectivo para Campylobacter Fernández, (1992a). En el laboratorio, estas fueron sembradas en placas con Agar Skirrow modificado Fernández, (1983) e incubadas bajo condiciones microaerófilas a 42 ?C durante 48 horas.

Después del periodo de incubación, las placas fueron examinadas a fin de determinar la presencia de presumibles colonias de Campylobacter spp. 
La identificación fue realizada, primero presuntivamente Luechtefeld \& Wang, (1981), utilizando las pruebas de la oxidasa y catalasa (ambas positivas) y las características macro, micromorfológicas y tintoriales observadas mediante la técnica de Gram (bacilos Gram negativos curvos en forma de "S"). Posteriormente, la identificación confirmativa de las especies de Campylobacter y de sus respectivos biovares se hizo de acuerdo al esquema propuesto por LIOR (1984).

\section{RESULTADOS}

Los resultados muestran que $92(36,8 \%)$ de las aves de corral estudiadas resultaron ser portadoras de las clásicas bacterias termotolerantes de Campylobacter, encontrándose la mayor tasa de aislamiento a nivel de pollos $(54,0 \%)$.

Los biovares I y II de $C$. jejuni ssp. jejuni fueron los más frecuentemente aislados $(12,0$ y $11,2 \%$, respectivamente) y se encontraron en todas las especies animales examinadas; C. coli también fue aislado a partir de todas las especies animales a excepción de palomas. C. lari fue aislado solamente a partir de pollos.

Las Tablas 1 y 2 muestran la distribución de las especies bacterianas en estudio y las de sus respectivos biovares, a nivel de la población avícola estudiada.

\section{Cuadro 1}

Frecuencia de aislamiento de las especies termotolerantes de

Campylobacter a partir de animales domésticos sanos.

\begin{tabular}{|c|c|c|c|c|}
\hline $\begin{array}{l}\text { Aves } \\
\text { estudiadas }\end{array}$ & $(n)$ & C.jejuni ssp. jejuni & C. coli & C. lari \\
\hline $\begin{array}{l}\text { Pollos } \\
10(10.0)\end{array}$ & 100 & $27(27,0)$ & & $17(17,0)$ \\
\hline $\begin{array}{l}\text { Pavos } \\
0(0.0)\end{array}$ & 50 & $15(30.0)$ & & $2(4.0)$ \\
\hline $\begin{array}{l}\text { Patos } \\
0(0.0)\end{array}$ & 50 & $9(18.0)$ & & $4(8.0)$ \\
\hline $\begin{array}{l}\text { Palomas } \\
0(0.0)\end{array}$ & 50 & $8(16.0)$ & & $0(0.0)$ \\
\hline $\begin{array}{l}\text { TOTAL } \\
10(4,0)\end{array}$ & 250 & $59(23,6)$ & & $23(9,2)$ \\
\hline
\end{tabular}




\section{Cuadro 2}

Distribución de biovares de las especies termotolerantes de Campylobacter aisladas de animales domésticos sanos.

\begin{tabular}{|c|c|c|c|c|c|c|c|c|c|c|}
\hline \multirow{2}{*}{$\begin{array}{l}\text { Aves } \\
\text { estudiadas }\end{array}$} & \multirow{2}{*}{5} & \multicolumn{4}{|c|}{ C. jejuni ssp. jejuni } & \multicolumn{2}{|c|}{ C. coli } & \multicolumn{2}{|c|}{ C. lari } & \multirow{2}{*}{$\mathrm{T} O \mathrm{O} \quad \mathrm{T}$} \\
\hline & & I & II & III & IV & I & II & I & II & \\
\hline Pollos & 100 & $16(16,0)$ & $10(10,0)$ & $1(1,0)$ & $0(0,0)$ & $5(5,0)$ & $12(12,0)$ & $6(6,0)$ & $4(4,0)$ & $54(54,0)$ \\
\hline Pavos & 50 & $9(18,0)$ & $6(12,0)$ & $0(0,0)$ & $0(0,0)$ & $0(0,0)$ & $2(4,0)$ & $0(0,0)$ & $0(0,0)$ & $17(34,0)$ \\
\hline Patos & 50 & $3(6,0)$ & $6(12,0)$ & $0(0,0)$ & $0(0,0)$ & $3(6,0)$ & $1(2,0)$ & $0(0,0)$ & $0(0,0)$ & $13(26,0)$ \\
\hline Palomas & 50 & $2(4,0)$ & $6(12,0)$ & $0(0,0)$ & $0(0,0)$ & $0(0,0)$ & $0(0,0)$ & $0(0,0)$ & $0(0,0)$ & $8(16,0)$ \\
\hline TOTAL & 250 & $30(12,0)$ & $28(11,2)$ & $(0,4)$ & ,0) & & $6,0)$ & $6(2,4)$ & $4(1,6)$ & $92(36,8)$ \\
\hline
\end{tabular}

()$=\%$

\section{DISCUSION}

En los resultados se aprecia un alto porcentaje de portación de estos agentes en pollos $(54,0 \%)$, valor que es inferior al encontrado por Figueroa et.al. (1982) en Chile (96,0\%), Fernández (1983) en Brasil $(86,8 \%)$, Shanker et.al. (1986) en Australia (74,0\%) y por Alvarez y Flores (1989) en México (95,0\%); pero es superior al encontrado por Lazarte y Pacheco (1986) en Cuzco-Perú (13,3\%) y Calnek (1991) en EE.UU. $(20,0 \%)$.

El alto porcentaje encontrado puede deberse probablemente a que en nuestro medio existen condiciones ambientales favorables para la propagación de estas bacterias como: alta temperatura, elevada humedad. Así mismo puede deberse a la carencia de medidas sanitarias, como la falta de redes de desagüe en zonas periurbanas, donde estas aves van con frecuencia en busca de alimento.

En cuanto a la portación encontrada en pavos (34,0\%), este valor es superior al encontrado por Kwiatek et.al. (1990) en Polonia (3,0\%). Este alto porcentaje también puede deberse a las mismas condiciones de riesgo señaladas para los pollos y patos; además existe otro factor a considerar, el contacto físico que se producen entre estas aves, ya que mayormente son criadas en forma agrupada.

Por su parte la portación encontrada en patos $(26,0 \%)$, es inferior a la obtenida por Kwiatek et.al. (1990) en Polonia $(48,0 \%)$, pero es superior a la encontrada por Grados et.al. (1988) en Lima-Perú $(18,2 \%)$. Al parecer este porcentaje significativo se debe a que la crianza de estas 
aves se lleva a cabo en forma libre y concurren con frecuencia a las instalaciones colectoras de aguas servidas donde permanecen muchas horas en busca de alimento; no hay que olvidar que estas aves poseen hábitos acuáticos, lo que hace que se encuentren en alto riesgo de contaminarse con estos agentes bacterianos, ya que existen reportes de haber sido encontrados en las plumas de estas aves (Rivera, 1990).

Referente al porcentaje de aislamiento obtenido en palomas, nuestro resultado $(16,0 \%)$ es aproximadamente similar al obtenido por Aguila y Cárdenas (1985) en Chile (17,4\%), inferior al encontrado en A lemania por Weber et.al. (1981) en palomas mensajeras (54,9\%), pero superior a los resultados obtenidos por Kapperud y Rosef (1983) en Noruega, quienes encontraron un porcentaje de positividad de 4,2\%.

En cuanto a las especies y biovares del género Campylobacter encontrados, podemos señalar que $C$. jejuni ssp.jejuni fue aislada en todas las especies de aves estudiadas, con una frecuencia del 23,6\%, siendo los biovares predominantes el I y II, lo que concuerda con Fernández (1988) y Lior (1984), quienes afirman que los biovares I y II son los más frecuentemente aislados a nivel mundial. Con respecto al biovar III, este sólo fue aislado de pollos con un bajo porcentaje $(0,4 \%)$; sin embargo, en las demás especies aviares no fue aislado este biovar, debido probablemente al menor número de muestras tomadas de cada uno de ellos. Con respecto al biovar IV de esta especie bacteriana, este no fue aislado en ninguna de las aves estudiadas, el cual es poco común (Kapperud y Rosef, 1983).

Por su parte $\mathrm{C}$. coli se aisló en todos los animales excepto en palomas; hallándose mayormente en pollos, en los que predominó el biovar II $(12,0 \%)$, lo que concuerda con los trabajos realizados por Rivera (1990) en muestras fecales de ovinos, donde el biovar de este agente bacteriano tiene el más alto índice de aislamiento en estos mamíferos.

En lo que respecta a la especie de $C$. lari, fue aislada únicamente de pollos $(10,0 \%)$, tanto el biovar I como el biovar II, lo que concuerda con los trabajos realizados por Skirrow \& Benjamin (1980) y por Kwiatek et.al. (1990) quienes aislaron C.lari a partir de gallinas y pollos, con una frecuencia relativamente baja. Al parecer, este porcentaje de aislamiento relativamente bajo podría deberse a que las especies aviares estudiadas no son el principal reservorio de C. lari Fernández, (1992b); TresierraA yala et.al., (1994), ya que su principal reservorio son las aves marinas, especialmente la gaviota del género Larus. 
La investigación realizada constituye una aproximación útil para conocer los aspectos relativos a la distribución de las bacterias termotolerantes de Campylobacter en las aves de corral de nuestro medio. La importante portación de Campylobacter spp., por parte de las aves estudiadas y el carácter zoonótico de la enfermedad producida por estas bacterias, pone de manifiesto la importancia de estas aves como reservorios y vehículos de transmisión de estos agentes, para el ser humano; lo cual hace necesario que su diagnóstico sea incorporado en forma rutinaria en los laboratorios de microbiología.

\section{AGRADECIMIENTOS}

Al Instituto de Investigaciones de la Facultad de Ciencias Biológicas (UNAP), así como al Consejo Nacional de Ciencia y Tecnología (CONCYTEC), por habernos brindado el apoyo económico para la realización de este proyecto.

Al Dr. Heriberto Fernández J aramillo de la Universidad Austral de Chile, por su asesoramiento técnico y científico.

\section{BIBLIOGRAFIA}

AGUILA, C. y CARDENAS, J. 1985. Aislamiento de Campylobacter termófilos en 6 especies de animales mediante el uso de cuatro sistemas de obtención de microaerofilia. Seminario de Titulación; Facultad de Medicina. Univ. Austral de Chile. pp.34.

ALVAREZ, V. and FLORES, J . 1989. Poultry as Campylobacter jejuni and Campylobacter coli: Contamination Source. Rev. Lat-amer. Microbiol. 31:271-273.

BLASER, M.; LA FORCE, M.; WILSON, N. and WANG, W. 1980. Reservoirs for human campylobacteriosis. J. Infect. Dis. 141:665-669.

BLASER, M. J .; TAYLOR, D. N. and FELDMAN, R. A. 1983. Epidemiology of Campylobacter jejuni infection. Rev. Epidemiol. 5:157-176.

CALNEK, B.1991. Diseases of poultry. 9?edition. Mc Graw Hill. U.S.A. 236244. 
FERNANDEZ, H. 1983. Especies Termófilas de Campylobacter: Aspectos bacteriológicos, epidemiológicos y patogénicos. Tesis de Doctorado. Escola Paulista de Medicina, Brazil. 54 pp.

1988. Species and biotypes distribution of thermolerant campylo bacters in animal reservoirs in southern Chile. Rev. Inst. Met. Trop. São Paulo 30: 357-360.

1992a. Increase of Campylobacter isolation rates using an enrichment medium. Rev. Microbiol. São Paulo. Brasil. 23: 5-7.

$1992 \mathrm{~b}$. Thermotolerant Campylobacter species associated with human diarrhoea in Latin America. J. Braz. Ass. Adv. Sci. 44 : 39-43.

FIGUEROA, G.; TOLEDO, M.; TRONCOSO, M ; \& SEPULVEDA, C. 1982. Aislamiento de Campylobacter fetus ssp.jejuni en pollos broiler. Rev. Chil. Nutr. 10: 87-98.

GRADOS,O.; BRAVO, N.; BLACK, N. and BUTZLER, J. P. 1988. Paedriatic Campylobacter diarrhoea from household exposure to live chickens in Lima, Perú. Boll. W. H. 0. 66 : 369374.

KAPPERUD, G. and ROSEF, O. 1983. Avian wildlife reservoir of Campylo bacter fetus spp.jejuni, Yersinia spp. and Salmonella spp. in Norway. Am. Soc. for Microbiol. 45: 375-380.

KWIATEK, K.; WOJ TON, B. and STERN, N. 1990. Prevalence and distribution of Campylobacter spp. in poultry and selected red meat carcasses in Poland. J. Food Prot. 53: 127-130.

LAZARTE, R. y PACHECO, R. 1986. Revisión del género Campylobacter y aislamiento de Campylobacter jejuni en muestras de pollos expendidos en el Cuzco. VIII Congreso Nacional de Biología. Libro de Resúmenes. A requipa-Perú. pg. 134.

LIOR, H. 1984. New extended biotyping scheme for Campylobacter jejuni, Campylobacter coli and "Campylobacter laridis". J. clin. Microbiol. 20: 636-640. 
LUECHTEFELD,W. \& WANG, W.L.L. 1981. Isolation of Campylobacter fetus subsp jejuni from zoo animals. J.A.V.M.A. 179: 11191122.

RIVERA, S.1990. Prevalencia de Campylobacter y riesgo de infección en una planta faenadora de carne. Tesis de Titulación; Facultad de Ciencias. Univ. Austral de Chile. 55 pp.

SHANKER, S.;LEE, A. and SORREL, T. 1986. Campylobacter jejuni in broiler: The role of vertical transmission. J. Hyg. Camb. 96: 153159.

SKIRROW, M. B. and BENJ AMIN, J. 1980. "1001" Campylobacters: Cultural characteristics of intestinal Campylobacters from man and animals. J. Hyg., 85: 427-442.

SMIBERT, R. 1984. Genus Campylobacter. En: KRIEG, N. \& HOLT, J . (eds). Bergey's Manual of Systematic Bacteriology. 9a. ed. Williams \& Wilkins. Baltimore. 111-117.

SOZA, G.; OSSA, G.; ILLESCA, V.; REYDET, P.; INOSTROZA, J. y RODRIGUEZ, J . 1987. Campylobacter jejuni en Diarrea Aguda del Lactante. Rev. Méd. Chile. 115:19-23.

TRESIERRA-AYALA, A.; BENDAYAN, M.E.; BERNUY, A.; PEREYRA, G. and FERNANDEZ, H. 1994. Chicken as Potential Contamination Source of Campylobacter lari in Iquitos, Peru. Rev.Inst.Med.Trop., São Paulo. 36:497-499.

VANDAMME, P. and DE LEY, J. 1991. Proposal for a new family Campylobacteriaceae. Int. J. Syst. Bacteriol. 41:451-455.

WEBER, A.; LEMBKE, C. and KETTNER, A. 1981. Nachweis von Campylobacter jejuni in kot proben von klinishc gesunden brief fauben. Berlín. Munich. Tierarztl. Wschr. 94: 449-451.

ZAMORA, J .; TRESIERRA, A.; ROJ AS, X.; REINHADTH, G. 1992. Biotipos de Campylobacter jejuni y Campylobacter coli aislados de bovinos. Arch. Med. Vet. 7: 65-68. 